\title{
On the moment distance of Poisson processes
}

\author{
Rafał Kapelko ${ }^{\mathrm{a}, *}$ \\ ${ }^{a}$ Department of Computer Science, Faculty of Fundamental Problems of Technology, Wroctaw University \\ of Science and Technology, Poland
}

\begin{abstract}
Consider the distance between two i.i.d. and independent Poisson processes with arrival rate $\lambda>0$ and respective arrival times $X_{1}, X_{2}, \ldots$ and $Y_{1}, Y_{2}, \ldots$ on a line. We give a closed analytical formula for the $\mathbf{E}\left[\left|X_{k+r}-Y_{k}\right|^{a}\right]$, for any integer $k \geq 1, r \geq 0$ and $a \geq 1$. The expected difference of the arrival times to the power $a$ between two i.i.d. and independent Poisson processes we represent as the combination of the Pochhammer polynomials.

Especially, for $r=0$ and any positive integer $a$, the following identity is valid$$
\mathbf{E}\left[\left|X_{k}-Y_{k}\right|^{a}\right]=\frac{a !}{\lambda^{a}} \frac{\Gamma\left(\frac{a}{2}+k\right)}{\Gamma(k) \Gamma\left(\frac{a}{2}+1\right)},
$$

where $\Gamma(z)$ is Gamma function.

Keywords: Poisson process, Moment distance, Gamma distribution

2010 MSC: 68R05, 60K30

\section{Introduction}

The cost of sensor movement has been the subject of interest in computer science research community. The paper [7] addresses the expected sum of movement of $n$ identical sensors displaced uniformly and independently at random in the unit interval to attain the coverage of the unit interval. Further, in [5] the authors studied the movement of $n$ sensors with identical $d$-dimensional cube sensing radius in $d$ dimensions when the cost of movement of sensor is proportional to some (fixed) power $a>0$.

Ajtai et al. [1] consider the optimal transportation cost for random matchings of bicolored point sets. The matching theorems for $N$ random variables independently uniformly distributed in the $d$-dimensional unit cube $[0,1]^{d}$, where $d \geq 2$ were investigated in the book [9].
\end{abstract}

${ }^{*}$ Corresponding author at: Department of Computer Science, Faculty of Fundamental Problems of Technology, Wrocław University of Science and Technology, Wybrzeże Wyspiańskiego 27, 50-370 Wrocław, Poland. Tel.: +48 7132033 62; fax: +48 713200751 .

Email address: rafal.kapelko@pwr.edu.pl (Rafał Kapelko)

${ }^{1}$ Research supported by grant $\mathrm{nr} 0401 / 0086 / 16$ 
More importantly, our work is closely related to [6] where the author studied the event distance between two i.i.d. and independent Poisson processes with arrival rate $\lambda>0$ and respective arrival times $X_{1}, X_{2}, \ldots$ and $Y_{1}, Y_{2}, \ldots$ on a line. In [6] the closed formula for the event distances $\mathbf{E}\left[\left|X_{k+r}-Y_{k}\right|\right]$, for any $k \geq 1, r \geq 0$ was derived as the combination of the Pochhammer polynomials. The following open problem was proposed in [6] to study more general moments $\mathbf{E}\left[\left|X_{i}-Y_{j}\right|^{a}\right]$, where $a$ is fixed.

We derive a closed formula for the moments $\mathbf{E}\left[\left|X_{k+r}-Y_{k}\right|^{a}\right]$, for any $k \geq 1, r \geq$ 0 , when $a$ is positive integer number.

\subsection{Preliminaries}

In this subsection we introduce some basic concepts and recall some useful identities involving indefinite and definite integrals, binomial coefficients and special functions which will be useful in the analysis in the next section.

We recall the definition of the Pochhammer polynomial [4]

$$
x^{(k)}= \begin{cases}x(x+1) \ldots(x+k-1) & \text { for } k \geq 1 \\ 1 & \text { for } k=0 .\end{cases}
$$

The Euler Gamma function $\Gamma(z)=\int_{0}^{\infty} t^{z-1} e^{-t} d t$ is defined for $z>0$. Moreover, we have

$$
\Gamma(n+1)=n !
$$

when $n$ is natural number.

We will use the Legendre duplication formula (see [8, Identity 5.5.5])

$$
\Gamma(2 z)=(2 \pi)^{-1 / 2} 2^{2 z-\frac{1}{2}} \Gamma(z) \Gamma\left(z+\frac{1}{2}\right) .
$$

Let $X_{i}$ be the arrival time of the $i$-th event in a Poisson process with arrival rate $\lambda>0$. We know that the random variable $X_{i}$ obeys the Gamma distribution with parameters $i \in \mathbb{N}_{+}, \lambda>0$. Its probability density function is given by

$$
f_{i, \lambda}(t)=\lambda e^{-\lambda t} \frac{(\lambda t)^{i-1}}{(i-1) !}
$$

and $\operatorname{Pr}\left[X_{i} \geq t\right]=\int_{t}^{\infty} f_{i, \lambda}(t) d t$. Notice that

$$
\int_{0}^{\infty} t^{k} f_{m, \lambda}(t) d t=\frac{m^{(k)}}{\lambda^{k}}
$$

where $m$ is nonnegative integer and $k \in \mathbb{N}$ (see [2, Chapter 8]). Using integration by parts we can derive the following identity

$$
\begin{aligned}
\int_{0}^{x} t^{k} f_{m, \lambda}(t) d t & =\left[-\frac{(m-1+k) !}{(m-1) ! \lambda^{k}} e^{-\lambda t} \sum_{l=0}^{m-1+k} \frac{(\lambda t)^{l}}{l !}\right]_{0}^{x} \\
& =\frac{m^{(k)}}{\lambda^{k}}\left(1-e^{-\lambda x} \sum_{l=0}^{m-1+k} \frac{(\lambda x)^{l}}{l !}\right)
\end{aligned}
$$


where $m$ is nonnegative integer, $k \in \mathbb{N}$ and $\lambda, x>0$.

We will use the following binomial identity

$$
\sum_{j=0}^{a}(-1)^{a-j}\left(\begin{array}{c}
j+k-1 \\
k-1
\end{array}\right)\left(\begin{array}{c}
a-j+k-1 \\
k-1
\end{array}\right)=\left\{\begin{array}{ll}
\frac{\Gamma\left(\frac{a}{2}+k\right)}{\Gamma(k) \Gamma\left(\frac{a}{2}+1\right)} & \text { if } a \equiv 0 \\
0 & \text { if } a \equiv 1
\end{array} \quad(\bmod 2) .\right.
$$

This identity can be checked using generating functions. Notice that

$$
\frac{1}{(1-z)^{k}}=\sum_{j \geq 0}\left(\begin{array}{c}
j+k-1 \\
k-1
\end{array}\right) z^{j}
$$

(see [4, Table 321, Section 7.2]). Combining Equation (7) with the elementary equality $\frac{1}{(1-z)^{k}} \frac{1}{(1+z)^{k}}=\frac{1}{\left(1-z^{2}\right)^{k}}$ we have

$$
\left(\sum_{j \geq 0}\left(\begin{array}{c}
j+k-1 \\
k-1
\end{array}\right) z^{j}\right)\left(\sum_{j \geq 0}\left(\begin{array}{c}
j+k-1 \\
k-1
\end{array}\right)(-1)^{j} z^{j}\right)=\sum_{j \geq 0}\left(\begin{array}{c}
j+k-1 \\
k-1
\end{array}\right) z^{2 j} .
$$

Multiplying together the power series in the left-hand side of Equation (8) and equating the coefficient of $z^{a}$ on both sides we get

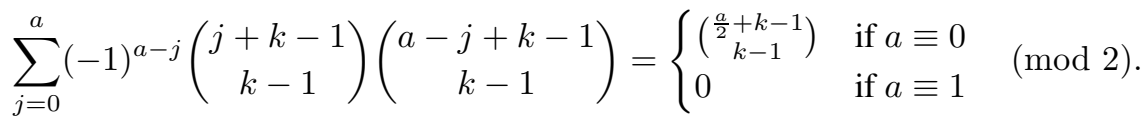

Note that, if $\frac{a}{2} \in \mathbb{N}_{+}$(see Equation (2) then

$$
\left(\begin{array}{c}
\frac{a}{2}+k-1 \\
k-1
\end{array}\right)=\frac{\left(\frac{a}{2}+k-1\right) !}{(k-1) !\left(\frac{a}{2}\right) !}=\frac{\Gamma\left(\frac{a}{2}+k\right)}{\Gamma(k) \Gamma\left(\frac{a}{2}+1\right)} .
$$

Putting together (9) and (10) we have the desired Formula (6).

Finally it is worth pointing out that, applying Formulas (4), (5), (6) in any mathematical software that performs symbolic calculation we get the expressions confirming these formulas. Let us consider Identity (4). We use the following command in Mathematica

Moment [ErlangDistribution [k, l], m]

and get the desired Identity (4)

$1^{\wedge}(-\mathrm{m})$ Pochhammer $[\mathrm{k}, \mathrm{m}]$.

For Identity (6) we apply the following command in Mathematica

$\operatorname{Sum}\left[(-1)^{\wedge}(a-j) \star B i n o m i a l[j+k-1, k-1]\right.$

$\star$ Binomial $[a-j+k-1, k-1],\{j, 0, a\}]$

and get

$\left(1+(-1)^{\wedge} a\right)$ Gamma $\left.[1 / 2(a+2 k)]\right) /(a \star G a m m a[a / 2] \star G a m m a[k])$.

It is easy to see that for $a \equiv 1(\bmod 2)$ Mathematica confirms Identity (6). When $a \equiv 0(\bmod 2)$ it is enough to combine together the output of Mathematica with equation $\Gamma\left[\frac{a}{2}+1\right]=\frac{a}{2} \Gamma\left[\frac{a}{2}\right]$ (see Equation (2) for $n:=\frac{a}{2}$ and $n:=\frac{a}{2}+1$ ). 


\subsection{Outline and results of the paper}

We consider the expected difference of the arrival times to the power $a$ between two i.i.d. and independent Poisson processes with arrival rate $\lambda>0$ and respective arrival times $X_{1}, X_{2}, \ldots$ and $Y_{1}, Y_{2}, \ldots$ on a line. We give a closed form formula $\mathbf{E}\left[\left|X_{k+r}-Y_{k}\right|^{a}\right]$, for any integer $k \geq 1, r \geq 0$, when $a$ is positive integer number as the combination of the Pochhammer polynomials (see Theorem 2 and Theorem 5 ).

Especially, for $r=0$, the closed analytical formula for $\mathbf{E}\left[\left|X_{k}-Y_{k}\right|^{a}\right]$, when $k \geq 1$ and $a \in \mathbb{N}_{+}$was obtained in terms of Gamma functions (see Theorem 2 and Theorem 7 ).

Here is an outline of the paper. In Section 2 we obtain closed formula for event distances to the power $a$ of two i.i.d. and independent Poisson processes, when $a \in \mathbb{N}_{+}$. Section 3 provides conclusions with some open problems.

\section{Main result}

Consider two i.i.d. and independent Poisson processes with arrival rate $\lambda>0$ and respective arrival times $X_{1}, X_{2}, \ldots$ and $Y_{1}, Y_{2}, \ldots$ on a line. We give a closed analytical formula for the moment distances $\mathbf{E}\left[\left|X_{k+r}-Y_{k}\right|^{a}\right]$, for any integer $k \geq$ $1, r \geq 0$, when $a$ is positive integer.

\subsection{Closed formula when a is a positive even natural number}

We begin with the following lemma which is helpful in the proof of Theorem 2 ,

Lemma 1. Assume that, $a$ is positive even natural number. Let $i \geq 1, k \geq 1$. Then

$$
\mathbf{E}\left[\left|X_{i}-Y_{k}\right|^{a}\right]=\frac{1}{\lambda^{a}} \sum_{j=0}^{a}\left(\begin{array}{l}
a \\
j
\end{array}\right)(-1)^{a-j} i^{(j)} k^{(a-j)} .
$$

Proof. As a first step, observe the following formula

$$
\mathbf{E}\left[\left|X_{i}-Y_{k}\right|^{a}\right]=\mathbf{E}\left[\left(X_{i}-Y_{k}\right)^{a}\right]=\sum_{j=0}^{a}\left(\begin{array}{l}
a \\
j
\end{array}\right)(-1)^{a-j} \mathbf{E}\left[X_{i}^{j}\right] \mathbf{E}\left[Y_{k}^{a-j}\right] .
$$

Applying Identity (4) for $m:=i, k:=j$ and $m:=k, k:=a-j$ as well as Definition (11) we deduce that

$$
\mathbf{E}\left[\left|X_{i}-Y_{k}\right|^{a}\right]=\sum_{j=0}^{a}\left(\begin{array}{l}
a \\
j
\end{array}\right)(-1)^{a-j} \frac{i^{(j)}}{\lambda^{j}} \frac{k^{(a-j)}}{\lambda^{a-j}}=\frac{1}{\lambda^{a}} \sum_{j=0}^{a}\left(\begin{array}{l}
a \\
j
\end{array}\right)(-1)^{a-j} i^{(j)} k^{(a-j)} .
$$

This completes the proof of Lemma 1

We are now ready to prove the main theorem, when $a$ is a positive even natural number. 
Theorem 2. Let a be a positive even natural number. Consider two i.i.d. and independent Poisson processes having identical arrival rate $\lambda>0$ and let $X_{1}, X_{2}, \ldots$ and $Y_{1}, Y_{2}, \ldots$ be their arrival times, respectively. The following identities are valid for all $k \geq 1, r \geq 0$

$$
\begin{aligned}
\mathbf{E}\left[\left|X_{k+r}-Y_{k}\right|^{a}\right] & =\frac{1}{\lambda^{a}} \sum_{j=0}^{a}\left(\begin{array}{l}
a \\
j
\end{array}\right)(-1)^{a-j}(k+r)^{(j)} k^{(a-j)}, \\
\mathbf{E}\left[\left|X_{k}-Y_{k}\right|^{a}\right] & =\frac{a !}{\lambda^{a}} \frac{\Gamma\left(\frac{a}{2}+k\right)}{\Gamma(k) \Gamma\left(\frac{a}{2}+1\right)} .
\end{aligned}
$$

Proof. The first part of the theorem follows immediately from Lemma1 with $i=k+r$. Putting together the first part of the theorem with $r=0$, Definition (11) and Identity (6) we get

$$
\begin{aligned}
\mathbf{E}\left[\left|X_{k}-Y_{k}\right|^{a}\right] & =\frac{1}{\lambda^{a}} \sum_{j=0}^{a}\left(\begin{array}{c}
a \\
j
\end{array}\right)(-1)^{a-j}(k)^{(j)} k^{(a-j)} \\
& =\frac{1}{\lambda^{a}} \sum_{j=0}^{a}\left(\begin{array}{c}
a \\
j
\end{array}\right)(-1)^{a-j} \frac{(k+j-1) !}{(k-1) !} \frac{(k+a-j-1) !}{(k-1) !} \\
& =\frac{a !}{\lambda^{a}} \sum_{j=0}^{a}(-1)^{a-j}\left(\begin{array}{c}
j+k-1 \\
k-1
\end{array}\right)\left(\begin{array}{c}
a-j+k-1 \\
k-1
\end{array}\right) \\
& =\frac{\Gamma\left(\frac{a}{2}+k\right)}{\Gamma(k) \Gamma\left(\frac{a}{2}+1\right)} .
\end{aligned}
$$

This is enough to prove Theorem 2

\subsection{Closed formula when a is an odd natural number}

It is worthwhile to mention that, when the parameter a in exponent is odd number, it is not so easy to derive the closed form formula (see Theorem 5 and Theorem 7 .

The general strategy of our proof is the following. In computing the moment $\mathbf{E}\left[\left|X_{i}-Y_{k}\right|^{a}\right]$, we are reduced to computing the moment $\mathbf{E}\left[\left|X_{i}-y_{k}\right|^{a}\right]$, where $y_{k}$ is fixed variable, $y_{k} \in(0, \infty)$ (see (12)).

Then we make an important observation that expectation $\mathbf{E}\left[\left|X_{i}-y_{k}\right|^{a}\right]$ is equal to the sum of the integrals (13) and (14). The first integral is easy to compute, while deriving the second integral is combinatorially challenging.

Our analysis of the moment distance proceeds along the following steps. Firstly, we give Lemma 3 and Lemma 4 which are helpful in the proof of Theorem 5 Then, Theorem 7 follows from Theorem 5 and Lemma 6 .

Lemma 3. The following identity is valid for all $i \geq 1, k \geq 1$, when a is an odd natural 
number

$$
\begin{aligned}
& \mathbf{E}\left[\left|X_{i}-Y_{k}\right|^{a}\right]=(-1) \frac{1}{\lambda^{a}} \sum_{j=0}^{a}\left(\begin{array}{l}
a \\
j
\end{array}\right)(-1)^{a-j} i^{(j)} k^{(a-j)} \\
& +\frac{1}{\lambda^{a}} \sum_{j=0}^{a}\left(\begin{array}{l}
a \\
j
\end{array}\right)(-1)^{a-j} i^{(j)} k^{(a-j)} \sum_{l=0}^{i+j-1}\left(\begin{array}{c}
k+l-1+a-j \\
l
\end{array}\right) \frac{1}{2^{k+l-1+a-j}} .
\end{aligned}
$$

Proof. As a first step, observe the following formula

$$
\begin{aligned}
\mathbf{E}\left[\left|X_{i}-Y_{k}\right|^{a}\right] & =\int_{0}^{\infty} \mathbf{E}\left[\left|X_{i}-y_{k}\right|^{a}\right] f_{k, \lambda}\left(y_{k}\right) d y_{k} \\
& =\int_{0}^{\infty} f_{k, \lambda}\left(y_{k}\right) \mathbf{E}\left[\left|X_{i}-y_{k}\right|^{a}\right] d y_{k} .
\end{aligned}
$$

Hence, computing the moment $\mathbf{E}\left[\left|X_{i}-Y_{k}\right|^{a}\right]$ is reduced to computing the moment $\mathbf{E}\left[\left|X_{i}-y_{k}\right|^{a}\right]$. Observe that

$$
\begin{aligned}
\mathbf{E}\left[\left|X_{i}-y_{k}\right|^{a}\right] & =\int_{y_{k}}^{\infty}\left(t-y_{k}\right)^{a} f_{i, \lambda}(t) d t+\int_{0}^{y_{k}}\left(y_{k}-t\right)^{a} f_{i, \lambda}(t) d t \\
& =\int_{0}^{\infty}\left(t-y_{k}\right)^{a} f_{i, \lambda}(t) d t-2 \int_{0}^{y_{k}}\left(t-y_{k}\right)^{a} f_{i, \lambda}(t) d t .
\end{aligned}
$$

Therefore, $\mathbf{E}\left[\left|X_{i}-Y_{k}\right|^{a}\right]$ is equal to the sum of the following two integrals which we evaluate separately.

$$
\begin{aligned}
& \int_{0}^{\infty} f_{k, \lambda}\left(y_{k}\right) \int_{0}^{\infty}\left(t-y_{k}\right)^{a} f_{i, \lambda}(t) d t d y_{k}, \\
& (-2) \int_{0}^{\infty} f_{k, \lambda}\left(y_{k}\right) \int_{0}^{y_{k}}\left(t-y_{k}\right)^{a} f_{i, \lambda}(t) d t d y_{k} .
\end{aligned}
$$

Case of integral (13).

Observe that

$$
\begin{aligned}
\int_{0}^{\infty} & f_{k, \lambda}\left(y_{k}\right) \int_{0}^{\infty}\left(t-y_{k}\right)^{a} f_{i, \lambda}(t) d t d y_{k} \\
& =\int_{0}^{\infty} f_{k, \lambda}\left(y_{k}\right) \mathbf{E}\left[\left|X_{i}-y_{k}\right|^{a}\right] d y_{k}=\mathbf{E}\left[\left(X_{i}-Y_{k}\right)^{a}\right] .
\end{aligned}
$$

After that, the calculation are almost exactly.

Applying Identity (4) and Definition (1) we have

$$
\int_{0}^{\infty} f_{k, \lambda}\left(y_{k}\right) \int_{0}^{\infty}\left(t-y_{k}\right)^{a} f_{i, \lambda}(t) d t d y_{k}=\frac{1}{\lambda^{a}} \sum_{j=0}^{a}\left(\begin{array}{l}
a \\
j
\end{array}\right)(-1)^{a-j} i^{(j)} k^{(a-j)} .
$$




\section{Case of integral (14).}

$$
\begin{aligned}
& (-2) \int_{0}^{\infty} f_{k, \lambda}\left(y_{k}\right) \int_{0}^{y_{k}}\left(t-y_{k}\right)^{a} f_{i, \lambda}(t) d t d y_{k} \\
& =(-2) \int_{0}^{\infty} f_{k, \lambda}\left(y_{k}\right) \int_{0}^{y_{k}} \sum_{j=0}^{a}\left(\begin{array}{l}
a \\
j
\end{array}\right)(-1)^{a-j} y_{k}^{a-j} t^{j} f_{i, \lambda}(t) d t d y_{k} \\
& =\sum_{j=0}^{a}\left(\begin{array}{l}
a \\
j
\end{array}\right)(-1)^{a-j} A(j),
\end{aligned}
$$

where

$$
A(j)=(-2) \int_{0}^{\infty} y_{k}^{a-j} f_{k, \lambda}\left(y_{k}\right) \int_{0}^{y_{k}} t^{j} f_{i, \lambda}(t) d t d y_{k} .
$$

Using Identity (5) for $x:=y_{k}, k:=j$ and $m:=i$ we get

$$
A(j)=(-2) \int_{0}^{\infty} y_{k}^{a-j} f_{k, \lambda}\left(y_{k}\right) \frac{i^{(j)}}{\lambda^{j}}\left(1-e^{-\lambda y_{k}} \sum_{l=0}^{i-1+j} \frac{\left(\lambda y_{k}\right)^{l}}{l !}\right) .
$$

Applying Identity (4) for $k:=a-j, m:=k$ and for $k:=0, m:=k-1+l+a-j$ $\lambda:=2 \lambda$ we have

$$
A(j)=A_{1}(j)+A_{2}(j),
$$

where

$$
\begin{aligned}
A_{1}(j) & =(-2) \int_{0}^{\infty} y_{k}^{a-j} f_{k, \lambda}\left(y_{k}\right) \frac{i^{(j)}}{\lambda^{j}} d y_{k}=(-2) \frac{i^{(j)}}{\lambda^{j}} \int_{0}^{\infty} y_{k}^{a-j} f_{k, \lambda}\left(y_{k}\right) d y_{k} \\
& =(-2) \frac{i^{(j)}}{\lambda^{j}} \frac{k^{(a-j)}}{\lambda^{a-j}}=\frac{(-2)}{\lambda^{a}} i^{(j)} k^{(a-j)} \\
A_{2}(j) & =\int_{0}^{\infty} 2 y_{k}^{a-j} f_{k, \lambda}\left(y_{k}\right) \frac{i^{(j)}}{\lambda^{j}} \sum_{l=0}^{i+j-1} e^{-\lambda y_{k}} \frac{\left(\lambda y_{k}\right)^{l}}{l !} d y_{k} \\
& =\frac{i^{(j)}}{\lambda^{a}} \sum_{l=0}^{i+j-1} \frac{1}{l !(k-1) ! 2^{k-1+l+a-j}} \int_{0}^{\infty} \frac{2 \lambda e^{-2 \lambda y_{k}}\left(2 \lambda y_{k}\right)^{k-1+l+a-j} d y_{k}}{\lambda^{a}} \sum_{l=0}^{i+j-1} \frac{1}{l !} \frac{(k-1+l+a-j) !}{(k-1) !} \frac{1}{2^{k+l-1+a-j}} \\
& =\frac{i^{(j)} k^{(a-j)}}{\lambda^{a}} \sum_{l=0}^{i+j-1}\left(\begin{array}{c}
k+a-j+l-1 \\
l
\end{array}\right) \frac{1}{2^{k+l-1+a-j}} .
\end{aligned}
$$

Therefore, we deduce that

$$
\sum_{j=0}^{a}\left(\begin{array}{l}
a \\
j
\end{array}\right)(-1)^{a-j} A_{1}(j)=(-2) \frac{1}{\lambda^{a}} \sum_{j=0}^{a}\left(\begin{array}{l}
a \\
j
\end{array}\right)(-1)^{a-j} i^{(j)} k^{(a-j)},
$$




$$
\begin{aligned}
\sum_{j=0}^{a}\left(\begin{array}{l}
a \\
j
\end{array}\right)(-1)^{a-j} A_{2}(j)= & \frac{1}{\lambda^{a}} \sum_{j=0}^{a}\left(\begin{array}{l}
a \\
j
\end{array}\right)(-1)^{a-j} i^{(j)} k^{(a-j)} \\
& \times \sum_{l=0}^{i+j-1}\left(\begin{array}{c}
k+l-1+a-j \\
l
\end{array}\right) \frac{1}{2^{k+l-1+a-j}} .
\end{aligned}
$$

Adding Formulas (15), (16) and (17) we derive the desired formula for $\mathbf{E}\left[\left|X_{i}-Y_{k}\right|^{a}\right]$, when $a$ is odd natural number. This completes the proof of Lemma 3 .

Now we give a simpler expression for the moment distance of two i.i.d. and independent Poisson processes in the following lemma.

Lemma 4. Assume that, $a$ is odd natural number. Let $i \geq 1, k \geq 1$. Then

$$
\begin{aligned}
\mathbf{E} & {\left[\left|X_{i}-Y_{k}\right|^{a}\right] } \\
& =\left(\sum_{l=k}^{i+a-1}\left(\begin{array}{c}
l+k-1 \\
l
\end{array}\right) \frac{1}{2^{l+k-1}}\right) \frac{1}{\lambda^{a}} \sum_{j=0}^{a}\left(\begin{array}{l}
a \\
j
\end{array}\right)(-1)^{a-j} i^{(j)} k^{(a-j)} \\
& +\frac{1}{\lambda^{a} 2^{i+k-2+a}} \sum_{l=0}^{a-1}\left(\sum_{j=0}^{l}\left(\begin{array}{c}
a \\
j
\end{array}\right)(-1)^{j} i^{(j)} k^{(a-j)}\right)\left(\begin{array}{c}
i+k+a-1 \\
i+l
\end{array}\right) .
\end{aligned}
$$

Proof. Applying Lemma 3 we deduce that

$$
\mathbf{E}\left[\left|X_{i}-Y_{k}\right|^{a}\right]=(-1) \frac{1}{\lambda^{a}} \sum_{j=0}^{a} B_{1}(j)+\frac{1}{\lambda^{a}} \sum_{j=0}^{a}\left(\begin{array}{l}
a \\
j
\end{array}\right)(-1)^{a-j} i^{(j)} k^{(a-j)} B_{2}(j) .
$$

where $B_{1}(j)=\left(\begin{array}{l}a \\ j\end{array}\right)(-1)^{a-j} i^{(j)} k^{(a-j)}$,

$$
B_{2}(j)=\sum_{l=0}^{i-1+j}\left(\begin{array}{c}
l+k-1+a-j \\
l
\end{array}\right) \frac{1}{2^{l+k-1+a-j}} .
$$

Using summation by parts

$\sum_{l=0}^{i-1+j} g(l+1)(f(l+1)-f(l))=\sum_{l=0}^{i+j}(g(l)-g(l+1)) f(l)+g(i+j+1) f(i+j)-g(0) f(0)$ for $f(l)=\frac{-2}{2^{l+k-1+a-j}}$ and $g(l)=\left\{\begin{array}{ll}\left(\begin{array}{ll}l-1+k-1+a-j & l-1\end{array}\right. & \text { for } l \geq 1 \\ 0 & \text { for } l=0\end{array}\right.$ as well as the following basic identity

$\left(\begin{array}{c}l+k-1+a-j \\ l\end{array}\right)-\left(\begin{array}{c}l-1+k-1+a-j \\ l-1\end{array}\right)=\left(\begin{array}{c}l+k-1+a-j-1 \\ l\end{array}\right)$ we have

$$
\begin{aligned}
& \sum_{l=0}^{i-1+j}\left(\begin{array}{c}
l+k-1+a-j \\
l
\end{array}\right) \frac{1}{2^{l+k-1+a-j}} \\
& =\sum_{l=0}^{i+j}\left(\begin{array}{c}
l+k-1+a-(j+1) \\
l
\end{array}\right) \frac{1}{2^{l+k-1+a-(j+1)}}-\frac{1}{2^{i+k-2+a}}\left(\begin{array}{c}
i+k+a-1 \\
i+j
\end{array}\right) .
\end{aligned}
$$


Therefore

$$
B_{2}(j)=B_{2}(j+1)+B_{3}(j)
$$

where

$$
B_{3}(j)=-\frac{1}{2^{i+k-2+a}}\left(\begin{array}{c}
i+k+a-1 \\
i+j
\end{array}\right) .
$$

Hence, we deduce that

$$
B_{2}(j)=B_{2}(a)+\sum_{l=j}^{a-1} B_{3}(l) \text { for } j \in\{0,1, \ldots, a-1\} .
$$

Applying Identity (21) to Formula (18) we have

$$
\begin{aligned}
\mathbf{E}\left[\left|X_{i}-Y_{k}\right|^{a}\right] & =\left(B_{2}(a)-1\right) \frac{1}{\lambda^{a}} \sum_{j=0}^{a}\left(\begin{array}{l}
a \\
j
\end{array}\right)(-1)^{a-j} i^{(j)} k^{(a-j)} \\
& +\frac{1}{\lambda^{a}} \sum_{j=0}^{a-1}\left(\begin{array}{l}
a \\
j
\end{array}\right)(-1)^{a-j} i^{(j)} k^{(a-j)} \sum_{l=j}^{a-1} B_{3}(l) .
\end{aligned}
$$

Using the identity $\sum_{i=0}^{m}\left(\begin{array}{c}m+j \\ m\end{array}\right) 2^{-j}=2^{m}$ (see [4, Identity 5.20, p. 167]) for $m:=k-1$ as well as Formula (19) we get

$$
\begin{aligned}
B_{2}(a)-1 & =\sum_{l=0}^{i+a-1}\left(\begin{array}{c}
l+k-1 \\
l
\end{array}\right) \frac{1}{2^{l+k-1}}-1 \\
& =\frac{1}{2^{k-1}}\left(\sum_{l=0}^{k-1}\left(\begin{array}{c}
l+k-1 \\
k-1
\end{array}\right) 2^{-l}+\sum_{l=k}^{i+a-1}\left(\begin{array}{c}
l+k-1 \\
l
\end{array}\right) 2^{-l}\right)-1 \\
& =\sum_{l=k}^{i+a-1}\left(\begin{array}{c}
l+k-1 \\
l
\end{array}\right) \frac{1}{2^{l+k-1}} .
\end{aligned}
$$

Finally, combining together (22), (23), (20) and changing summation in the second sum in 22) we get the desired result.

We are now ready to give the first main result, when $a$ is an odd natural number.

Theorem 5. Let a be an odd natural number. Consider two i.i.d. and independent Poisson processes having identical arrival rate $\lambda>0$ and let $X_{1}, X_{2}, \ldots$ and $Y_{1}, Y_{2}, \ldots$ be their arrival times, respectively. The following identity is valid for all $r \geq 0, k \geq 1$

$$
\begin{aligned}
\mathbf{E} & {\left[\left|X_{k+r}-Y_{k}\right|^{a}\right] } \\
& =\frac{1}{\lambda^{a}} \frac{\Gamma\left(k+\frac{1}{2}\right)}{\Gamma\left(\frac{1}{2}\right) \Gamma(k+1)} \sum_{l=0}^{r+a-1} \frac{(2 k)^{(l)}}{(k+1)^{(l)} 2^{l}} \sum_{j=0}^{a}\left(\begin{array}{l}
a \\
j
\end{array}\right)(-1)^{a-j}(k+r)^{(j)} k^{(a-j)} \\
& +\frac{1}{\lambda^{a} 2^{r-1}} \frac{\Gamma\left(\frac{a}{2}+k\right)}{\Gamma(1 / 2) \Gamma(k)} \sum_{l=0}^{a-1}\left(\sum_{j=0}^{l}\left(\begin{array}{l}
a \\
j
\end{array}\right)(-1)^{j}(k+r)^{(j)} k^{(a-j)}\right) \frac{k^{\left(\frac{a+1}{2}\right)(2 k+a)^{(r)}}}{k^{(r+l+1)} k^{(a-l)}} .
\end{aligned}
$$


Proof. Applying Lemma4 for $i=k+r$ we deduce that

$$
\begin{aligned}
\mathbf{E}\left[\left|X_{k+r}-Y_{k}\right|^{a}\right] & =\frac{1}{\lambda^{a}} \sum_{l=k}^{k+r+a-1}\left(\begin{array}{c}
l+k-1 \\
l
\end{array}\right) \frac{1}{2^{l+k-1}} C(k, r, a) \\
& +\frac{1}{\lambda^{a} 2^{2 k+r-2+a}} \sum_{l=0}^{a-1} C(k, r, l)\left(\begin{array}{c}
2 k+r+a-1 \\
k+r+l
\end{array}\right),
\end{aligned}
$$

where

$$
C(k, r, l)=\sum_{j=0}^{l}\left(\begin{array}{l}
a \\
j
\end{array}\right)(-1)^{j}(k+r)^{(j)} k^{(a-j)} .
$$

Using the Legendre duplication formula (3) for $z=\frac{a-1}{2}+k$ we get

$$
\Gamma(2 k+a-1)=\pi^{-1 / 2} 2^{2 k+a-2} \Gamma\left(\frac{a-1}{2}+k\right) \Gamma\left(\frac{a}{2}+k\right) .
$$

Applying Formula (26) for $a=1$ and the identity $\Gamma(1 / 2)=\sqrt{\pi}$ as well as Equation (2) we derive

$$
2^{-2 k+1} \frac{(2 k-1) !}{(k-1) ! k !}=\frac{\Gamma\left(k+\frac{1}{2}\right)}{\Gamma\left(\frac{1}{2}\right) \Gamma(k+1)} .
$$

Using this and Definition (1) we have

$$
\begin{aligned}
& \sum_{l=k}^{k+r+a-1}\left(\begin{array}{c}
l+k-1 \\
l
\end{array}\right) \frac{1}{2^{l+k-1}}=\frac{1}{2^{2 k-1}} \sum_{l=0}^{r+a-1}\left(\begin{array}{c}
2 k-1+l \\
k+l
\end{array}\right) \frac{1}{2^{l}} \\
= & 2^{-2 k+1} \sum_{l=0}^{r+a-1} \frac{(2 k-1) !(2 k)^{(l)}}{(k-1) ! k !(k+1)^{(l)} 2^{l}}=\frac{\Gamma\left(k+\frac{1}{2}\right)}{\Gamma\left(\frac{1}{2}\right) \Gamma(k+1)} \sum_{l=0}^{r+a-1} \frac{(2 k)^{(l)}}{(k+1)^{(l)} 2^{l}} .
\end{aligned}
$$

Combining Definition (1), Equation (2), the identity $\Gamma(1 / 2)=\sqrt{\pi}$ and Formula (26) we get

$$
\begin{gathered}
\frac{1}{2^{2 k+r-2+a}}\left(\begin{array}{c}
2 k+r+a-1 \\
k+r+l
\end{array}\right)=\frac{1}{2^{2 k+r-2+a}} \frac{(2 k+r+a-1) !}{(k+r+l) !(k+a-1-l) !} \\
=\frac{1}{2^{2 k+r-2+a}} \frac{\Gamma(2 k+a-1)(2 k+a-1)(2 k+a)^{(r)}}{\Gamma(k) k^{(r+l+1)}(k-1) ! k^{(a-l)}} \\
=\frac{\Gamma\left(\frac{a}{2}+k\right)}{\Gamma(1 / 2) \Gamma(k)} \frac{k^{\left(\frac{a+1}{2}\right)}}{2^{r-1}} \frac{(2 k+a)^{(r)}}{k^{(r+l+1)} k^{(a-l)}} .
\end{gathered}
$$

Putting together (24), (25), (27) and (28) completes the proof of Theorem 5 ,

The next lemma will be helpful in the proof of Theorem 7

The proof of Lemma 6 is technically complicated and the overall strategy is the following. Firstly, we define $D(k, a)$ by Formula (32). Combing together Identities (6) 
and $k^{(a-j)} k^{(j)}\left(\begin{array}{c}a \\ j\end{array}\right)=k^{(j)} k^{(a-j)}\left(\begin{array}{c}a \\ a-j\end{array}\right)$ we represent $D(k, a)$ by Formula 33 . Then we make an important observation that $D(k, a)$ represented by Equation (33) is the polynomial of variable $k$ of degree less than or equal to $\frac{a-1}{2}$. We prove that all coefficient of $D(k, a)$ expect constant term are equal to zero. Using the binomial identities (34), (35) and the Legendre duplication formula (3) we deduce that

$$
D(k, a)=\frac{a ! \sqrt{\pi}}{2 \Gamma\left(\frac{a}{2}+1\right)} \text { for each } k \in\left\{0,-1,-2 \ldots,-\frac{a-1}{2}\right\} .
$$

Therefore, Identity (29) is enough to prove that $D(k, a)$ is constant equal to $\frac{a ! \sqrt{\pi}}{2 \Gamma\left(\frac{a}{2}+1\right)}$.

The following Mathematica code can be used to confirm numerically validity of Lemma (6)

$$
\begin{aligned}
& \mathrm{F}\left[\mathrm{a}_{-}\right]:=\operatorname{Sum}\left[\operatorname { S u m } \left[(-1)^{\wedge} \mathrm{j} * \operatorname{Pochhammer}[\mathrm{k}, \mathrm{a}-\mathrm{j}] * \operatorname{Pochhammer}[\mathrm{k}, \mathrm{j}]\right.\right. \\
& * \text { Binomial }[\mathrm{a}, \mathrm{j}],\{\mathrm{j}, 0,1\}] * \operatorname{Pochhammer}[\mathrm{k},(\mathrm{a}+1) / 2] \\
& \left.*(\operatorname{Pochhammer}[\mathrm{k}, 1+1] * \operatorname{Pochhammer}[\mathrm{k}, \mathrm{a}-1])^{\wedge}(-1),\{1,0, \mathrm{a}-1\}\right] \\
& -\left(\operatorname{Gamma}[\mathrm{a}+1] * \operatorname{Pi}^{\wedge}(1 / 2)\right) /(2 * \operatorname{Gamma}[\mathrm{a} / 2+1])
\end{aligned}
$$

Then the following command

\section{FullSimplify [F[a]]}

gives zero for fixed odd natural parameter $a$.

Lemma 6. Assume that, $a$ is an odd natural number. Let $k \geq 1$. Then

$$
\sum_{l=0}^{a-1}\left(\sum_{j=0}^{l}(-1)^{j} k^{(a-j)} k^{(j)}\left(\begin{array}{l}
a \\
j
\end{array}\right)\right) \frac{k^{\left(\frac{a+1}{2}\right)}}{k^{(1+l)} k^{(a-l)}}=\frac{a ! \sqrt{\pi}}{2 \Gamma\left(\frac{a}{2}+1\right)} .
$$

Proof. From Identities (6) and $k^{(a-j)} k^{(j)}\left(\begin{array}{l}a \\ j\end{array}\right)=k^{(j)} k^{(a-j)}\left(\begin{array}{c}a \\ a-j\end{array}\right)$ we deduce that

$$
\begin{aligned}
\sum_{j=0}^{l}(-1)^{j} k^{(a-j)} k^{(j)}\left(\begin{array}{c}
a \\
j
\end{array}\right) & =-\sum_{j=a}^{a-l} k^{(j)} k^{(a-j)}(-1)^{j}\left(\begin{array}{c}
a \\
a-j
\end{array}\right) \\
=\sum_{j=0}^{a-l-1} k^{(j)} k^{(a-j)}(-1)^{j}\left(\begin{array}{c}
a \\
a-j
\end{array}\right) & =\sum_{j=0}^{a-l-1}(-1)^{j} k^{(a-j)} k^{(j)}\left(\begin{array}{c}
a \\
j
\end{array}\right) .
\end{aligned}
$$

Let

$$
D(k, a):=\sum_{l=0}^{a-1}\left(\sum_{j=0}^{l}(-1)^{j} k^{(a-j)} k^{(j)}\left(\begin{array}{l}
a \\
j
\end{array}\right)\right) \frac{k^{\left(\frac{a+1}{2}\right)}}{k^{(1+l)} k^{(a-l)}} .
$$

Applying Equation (31) we deduce that

$$
D(k, a)=D_{1}(k, a)+D_{2}(k, a)
$$


where

$$
\begin{aligned}
& D_{1}(k, a)=\sum_{l=0}^{\frac{a-1}{2}}\left(\sum_{j=0}^{l}(-1)^{j} k^{(a-j)} k^{(j)}\left(\begin{array}{l}
a \\
j
\end{array}\right)\right) \frac{k^{\left(\frac{a+1}{2}\right)}}{k^{(1+l)} k^{(a-l)}}, \\
& D_{2}(k, a)=\sum_{l=\frac{a-1}{2}+1}^{a-1}\left(\sum_{j=0}^{a-l-1}(-1)^{j} k^{(a-j)} k^{(j)}\left(\begin{array}{l}
a \\
j
\end{array}\right)\right) \frac{k^{\left(\frac{a+1}{2}\right)}}{k^{(1+l)} k^{(a-l)}} .
\end{aligned}
$$

Therefore, we have

$$
\begin{aligned}
D(k, a) & =\sum_{l=0}^{\frac{a-1}{2}} \sum_{j=0}^{l}\left(\begin{array}{l}
a \\
j
\end{array}\right)(-1)^{j} k^{(j)}(k+a-l)^{(l-j)}(k+l+1)^{\left(\frac{a-1}{2}-l\right)} \\
& +\sum_{l=\frac{a-1}{2}+1}^{a-1} \sum_{j=0}^{a-l-1}\left(\begin{array}{l}
a \\
j
\end{array}\right)(-1)^{j} k^{(j)}(k+l+1)^{(a-1-j-l)}(k+a-l)^{\left(l-\frac{a-1}{2}\right) .}
\end{aligned}
$$

Observe that $(k+a-l)^{(l-j)},(k+l+1)^{\left(\frac{a-1}{2}-l\right)}$ are polynomials of variable $k$ for each $j \in\{0,1, \ldots, l\}, l \in\left\{0,1, \ldots, \frac{a-1}{2}\right\}$ and $(k+l+1)^{(a-1-j-l)},(k+a-l)^{\left(l-\frac{a-1}{2}\right)}$ are polynomials of variable $k$ for each $j \in\{0,1, \ldots, a-1-l\}, l \in\left\{\frac{a-1}{2}+1, \ldots, a-1\right\}$.

Therefore, $D(k, a)$ can be only the polynomial of variable $k$ of degree less than or equal to $\frac{a-1}{2}$.

We have to prove that for all $k \in \mathbb{N}_{+}: D(k, a)$ is constant equal to $\frac{a ! \sqrt{\pi}}{2 \Gamma\left(\frac{a}{2}+1\right)}$.

Hence, to prove Equality (30) it remains to obtain the following equality

$$
\begin{aligned}
D(k, a) & =\sum_{l=0}^{\frac{a-1}{2}} \sum_{j=0}^{l}\left(\begin{array}{l}
a \\
j
\end{array}\right)(-1)^{j} k^{(j)}(k+a-l)^{(l-j)}(k+l+1)^{\left(\frac{a-1}{2}-l\right)} \\
& +\sum_{l=\frac{a-1}{2}+1}^{a-1} \sum_{j=0}^{a-l-1}\left(\begin{array}{l}
a \\
j
\end{array}\right)(-1)^{j} k^{(j)}(k+l+1)^{(a-1-j-l)}(k+a-l)^{\left(l-\frac{a-1}{2}\right)} \\
& =\frac{a ! \sqrt{\pi}}{2 \Gamma\left(\frac{a}{2}+1\right)} \text { for each } k=0,-1,-2, \ldots,-\frac{a-1}{2} .
\end{aligned}
$$

Let $b \in\left\{0, \ldots, \frac{a-1}{2}\right\}$. Observe that

$$
\begin{gathered}
(-b+a-l)^{(l-j)}(-b+l+1)^{\left(\frac{a-1}{2}-l\right)}=0 \text { for } 0 \leq l \leq b-1,0 \leq j \leq l, \\
(-b+l+1)^{(a-1-j-l)}(-b+a-l)^{\left(l-\frac{a-1}{2}\right)}=0 \text { for } a-1-(b-1) \leq l \leq a-1, \\
0 \leq j \leq a-l-1 \\
(-b)^{(j)}=0 \text { for } b+1 \leq j .
\end{gathered}
$$


Applying this we have

$$
\begin{aligned}
D(-b, a) & =\sum_{l=b}^{\frac{a-1}{2}} \sum_{j=0}^{b}\left(\begin{array}{l}
a \\
j
\end{array}\right)(-1)^{j}(-b)^{j}(-b+a-l)^{(l-j)}(-b+l+1)^{\left(\frac{a-1}{2}-l\right)} \\
& +\sum_{l=\frac{a-1}{2}+1}^{a-1-b} \sum_{j=0}^{b}\left(\begin{array}{l}
a \\
j
\end{array}\right)(-1)^{j}(-b)^{j}(-b+l+1)^{(a-1-j-l)}(-b+a-l)^{\left(l-\frac{a-1}{2}\right)} \\
& =\sum_{l=b}^{\frac{a-1}{2}} \sum_{j=0}^{b}\left(\begin{array}{l}
a \\
j
\end{array}\right) \frac{b !}{(b-j) !} \frac{(a-j-1-b) !}{(a-l-1-b) !} \frac{\left(\frac{a-1}{2}-b\right) !}{(l-b) !} \\
& +\sum_{l=\frac{a-1}{2}+1}^{a-1-b} \sum_{j=0}^{b}\left(\begin{array}{l}
a \\
j
\end{array}\right) \frac{b !}{(b-j) !} \frac{(a-j-1-b) !}{(l-b) !} \frac{\left(\frac{a-1}{2}-b\right) !}{(a-l-1-b) !} \\
= & \left(\frac{a-1}{2}-b\right) ! b !\left(\sum_{j=0}^{b}\left(\begin{array}{l}
a \\
j
\end{array}\right)\left(\begin{array}{c}
a-1-b-j \\
b-j
\end{array}\right)\right) \sum_{l=b}^{a-1-b}\left(\begin{array}{c}
a-1-2 b \\
a-l-1-b
\end{array}\right) .
\end{aligned}
$$

Notice that

$$
\sum_{l=b}^{a-1-b}\left(\begin{array}{c}
a-1-2 b \\
a-l-1-b
\end{array}\right)=\sum_{l=0}^{a-1-2 b}\left(\begin{array}{c}
a-1-2 b \\
l
\end{array}\right)=2^{a-2 b-1} .
$$

Applying this and the identity

$$
\sum_{j=0}^{b}\left(\begin{array}{l}
a \\
j
\end{array}\right)\left(\begin{array}{cl}
a-1-b-j \\
b-j
\end{array}\right)= \begin{cases}\frac{2^{b}}{b !} \prod_{j=1}^{b}(a-(2 j-1)) & \text { if } b \neq 0 \\
1 & \text { if } b=0 .\end{cases}
$$

(see [3, Identity 7.17, p. 36]) we get

$$
\begin{aligned}
D(-b, a) & =2^{a-1}\left(\frac{a-1}{2}-b\right) ! \begin{cases}\frac{2^{b}}{b !} \prod_{j=1}^{b}(a-(2 j-1)) & \text { if } b \neq 0 \\
1 & \text { if } b=0\end{cases} \\
& =2^{a-1}\left(\frac{a-1}{2}\right) !
\end{aligned}
$$

Finally, from the Legendre duplication formula (3) for $z=\frac{a+1}{2}$ we deduce that

$$
D(-b, a)=\frac{a ! \sqrt{\pi}}{2 \Gamma\left(\frac{a}{2}+1\right)} \text { for all } b \in\left\{0, \ldots, \frac{a-1}{2}\right\} .
$$

This is enough to prove Lemma 6

Finally, we can prove the second main result of this subsection. 
Theorem 7. Let a be an odd natural number. Consider two i.i.d. and independent Poisson processes having identical arrival rate $\lambda>0$ and let $X_{1}, X_{2}, \ldots$ and $Y_{1}, Y_{2}, \ldots$ be their arrival times, respectively. The following identity is valid for all $k \geq 1$ :

$$
\mathbf{E}\left[\left|X_{k}-Y_{k}\right|^{a}\right]=\frac{a !}{\lambda^{a}} \frac{\Gamma\left(\frac{a}{2}+k\right)}{\Gamma(k) \Gamma\left(\frac{a}{2}+1\right)} .
$$

Proof. Firstly, combining together Definition (1) and Identity (6) we deduce that

$$
\sum_{j=0}^{a}\left(\begin{array}{l}
a \\
j
\end{array}\right)(-1)^{a-j} k^{(j)} k^{(a-j)}=a ! \sum_{j=0}^{a}(-1)^{a-j}\left(\begin{array}{c}
j+k-1 \\
k-1
\end{array}\right)\left(\begin{array}{c}
a-j+k-1 \\
k-1
\end{array}\right)=0 .
$$

We substitute Equation (36) into Theorem 5 and get

$$
\begin{aligned}
& \mathbf{E}\left[\left|X_{k}-Y_{k}\right|^{a}\right] \\
& =\frac{1}{\lambda^{a} \Gamma(1 / 2) 2^{-1}} \frac{\Gamma\left(\frac{a}{2}+k\right)}{\Gamma(k)} \sum_{l=0}^{a-1}\left(\sum_{j=0}^{l}\left(\begin{array}{l}
a \\
j
\end{array}\right)(-1)^{j}(k)^{(j)} k^{(a-j)}\right) \frac{k^{\left(\frac{a+1}{2}\right)}}{k^{(l+1)} k^{(a-l)}} .
\end{aligned}
$$

Finally, the result of Theorem 7 follows from Lemma 6 and the identity $\Gamma(1 / 2)=$ $\sqrt{\pi}$.

\section{Conclusion}

In this paper, we studied the expected difference of the arrival times to the power $a$ between Poisson events of two i.i.d. and independent Poisson processes with arrival rate $\lambda>0$ and respective arrival times $X_{1}, X_{2}, \ldots$ and $Y_{1}, Y_{2}, \ldots$ on a line.

It is obtained a closed form formula for the $\mathbf{E}\left[\left|X_{k+r}-Y_{k}\right|^{a}\right]$, where $k \geq 1, r \geq 0$ and $a \in \mathbb{N}_{+}$. as the combination of the Pochhammer polynomials. Especially, for $r=0$ and any positive integer $a, \mathbf{E}\left[\left|X_{k}-Y_{k}\right|^{a}\right]=\frac{a !}{\lambda^{a}} \frac{\Gamma\left(\frac{a}{2}+k\right)}{\Gamma(k) \Gamma\left(\frac{a}{2}+1\right)}$, where $\Gamma(z)$ is Gamma function.

It is worthwhile to mention that, when $a$ is odd, it is combinatorially challenging to derive the closed form formula. For $a$ even it is unsurprisingly easier to obtain the closed form formula.

It would be interesting for future research to find the closed form formula for the expected difference to the power $a$ of two identical other more general random variables in (1) dimension as well as (2) in the higher dimension.

\section{References}

[1] M. Ajtai, J. Komlós, and G. Tusnády. On optimal matchings. Combinatorica, 4(4):259-264, 1984.

[2] Evans M. Hastings N. Peacock B. Forbes, C. Statistical Distributions. John Wiley \& Sons, 2011. 
[3] H. Gould and J. Quaintance. Tables of Combinatorial Identities, volume 4. http://www.math.wvu.edu/ gould/Vol.4.PDF.

[4] R. Graham, D. Knuth, and O. Patashnik. Concrete Mathematics A Foundation for Computer Science. Addison-Wesley, Reading, MA, 1994.

[5] R. Kapelko and E. Kranakis. On the displacement for covering a d-dimensional cube with randomly placed sensors. Ad Hoc Networks, 40:37-45, 2016.

[6] E. Kranakis. On the event distance of poisson processes with applications to sensors. Discrete Applied Mathematics, 179:152 - 162, 2014.

[7] E. Kranakis, D. Krizanc, O. Morales-Ponce, L. Narayanan, J. Opatrny, and S. Shende. Expected sum and maximum of displacement of random sensors for coverage of a domain. In Proceedings of the 25th ACM symposium on Parallelism in algorithms and architectures, pages 73-82. ACM, 2013.

[8] NIST Digital Library of Mathematical Functions. http://dlmf.nist.gov/8.17.

[9] M. Talagrand. Upper and Lower Bounds for Stochastic Processes. Springer, 2014. 